'Servicio de Medicina Interna, Hospital San Pablo. Coquimbo, Chile.

2Unidad de Hemodiálisis y Plasmaféresis, Hospital San Pablo. Coquimbo, Chile.

${ }^{3} U$ nidad de Tratamiento Intermedio, Hospital San Pablo, Coquimbo. Chile.

Trabajo no recibió financiamiento. Los autores declaran no tener conflictos de interés.

Recibido el 13 de mayo de 2020, aceptado el 26 de agosto de 2020.

Correspondencia a Camila Jure Bustamante Avenida Videla S/N. Coquimbo, Chile. camila.jure.b@gmail.com

\section{Hipertrigliceridemia severa refractaria a tratamiento médico y pancreatitis aguda recurrente: uso de plasmaféresis crónica ambulatoria en el Hospital San Pablo de Coquimbo. Reporte de un caso}

\author{
CAMILA JURE B. ${ }^{1}$, LUCY SAPIAIN P. ${ }^{1,2}$, SUSANA GONZÁLEZ F. ${ }^{3}$
}

\begin{abstract}
Severe Hypertriglyceridemia (HTG) is associated with complications such as acute pancreatitis (AP) with high morbidity and mortality rates. We report a 42 years-old man with refractory HTG diagnosed at 19 years of age, and multiple episodes of AP, admitted with the suspicion of a new AP episode. Serum triglycerides were over $2000 \mathrm{mg} / \mathrm{dl}$. His body mass index was $18 \mathrm{~kg} / \mathrm{m}^{2}$, there was no evidence of xanthomas or xanthelasmas, but lipemia retinalis was found. Management included heparin and insulin, added to his usual treatment with fibrates, statins, omega-3 fatty acids, and orlistat. Due to lack of response, apheresis was started. After five sessions, triglycerides decreased to $588 \mathrm{mg} / \mathrm{dl}$ ( $82 \%$ reduction) and levels remained below $1000 \mathrm{mg} / \mathrm{dl}$ with daily apheresis. The patient continued with weekly sessions as outpatient with a sustained good response.
\end{abstract}

(Rev Med Chile 2020; 148: 1362-1367)

Key words: Dyslipidemias; Fibric Acids; Pancreatitis; Plasmapheresis; Triglycerides.

\footnotetext{
L
} a hipertrigliceridemia (HTG) es una condición prevalente que continúa en aumento ${ }^{1}$, definida como niveles de triglicéridos séricos sobre el percentil 95 según edad y sexo para una población, aunque la definición exacta varía entre grupos de expertos ${ }^{2}$. Generalmente, se clasifica como "primaria" cuando se sospecha origen familiar o hereditario, y "secundaria" a los casos donde coexisten uno o más factores identificables como síndrome metabólico, consumo de alcohol o medicamentos ${ }^{3}$. Actualmente, gracias a estudios de asociación del genoma, se ha evidenciado que en casos de hipertrligliceridemia severa probablemente existe una base genética (monogénica y mayormente poligénica) asociada a condiciones secundarias, por lo que parece más adecuado definir los síndromes de hipertrigliceridemia de acuerdo a su etiología genética ${ }^{4,5}$.

La hipertrigliceridemia severa es una de las principales causas de pancreatitis aguda (PA), alcanzando hasta $10 \%$ de los $\operatorname{casos}^{6,7}$. Aunque la mayoría de los casos son leves, los severos se asocian a altas tasas de mortalidad, de hasta $30 \%{ }^{8}$. Habitualmente se describe que con TG séricos mayores a $1.000 \mathrm{mg} / \mathrm{dl}$ se puede desencadenar PA y sus complicaciones ${ }^{9}$. Este rango es arbitrario y el umbral sobre el cual la PA pudiera ocurrir es actualmente desconocido. Por tanto, la reducción rápida y sostenida de los niveles de triglicéridos es un objetivo primario en la prevención de complicaciones asociadas a esta entidad.

El manejo general de estos pacientes incluye 
el tratamiento de la condición aguda y medidas de mantención, como modificaciones dietéticas y fármacos ${ }^{10}$. En aquellos casos refractarios, la aféresis precoz es una opción interesante a considerar y se ha utilizado de manera exitosa en pacientes con PA por HTG severa, lo que no sólo ha sido confirmado en varios estudios, sino también sugerido por la Sociedad Americana de Aféresis ${ }^{11}$. Prácticamente no existe experiencia en el uso de esta medida como tratamiento crónico para prevenir el desarrollo de complicaciones.

Se presenta el caso de un paciente con hipertrigliceridemia refractaria a tratamiento médico, con antecedente de múltiples pancreatitis agudas que se mantiene con plasmaféresis crónica con adecuada respuesta a la fecha. Se obtiene consentimiento informado para su publicación.

\section{Presentación del caso}

Hombre de 42 años, con antecedente de hipertrigliceridemia diagnosticada a los 19 años, cuadros de dolor abdominal recurrente y múltiples hospitalizaciones en contexto de PA (11 episodios objetivados). En tratamiento con varios esquemas que incluyen estatinas, fibratos, omega-3; incluso habría recibido insulina glargina de manera ambulatoria sin tener diagnóstico de diabetes. Pese a ello, mantenía triglicéridos $>2.000 \mathrm{mg} / \mathrm{dl}$. En diciembre de 2017 se diagnosticó trombosis de vena mesentérica superior secundaria a estenosis parcial por lesión compleja de cabeza de páncreas, cuyo estudio concluyó pseudoquiste pancreático. Inició tratamiento anticoagulante con acenocumarol y se ajustó terapia de hipertrigliceridemia a ciprofibrato $200 \mathrm{mg} /$ día y orlistat $120 \mathrm{mg} 3$ veces al día. Requirió cambio de tratamiento anticoagulante ya que hipertrigliceridemia interfería en controles de INR.

En octubre de 2018 se hospitalizó en el Hospital de Coquimbo en bajo sospecha diagnóstica de $\mathrm{PA}$, con triglicéridos en $2.035 \mathrm{mg} / \mathrm{dl}$. Al examen físico, IMC $18 \mathrm{~kg} / \mathrm{m}^{2}$, sin evidencia de xantomas o xantelasmas, pero se confirmó lipemia retinalis a la evaluación oftalmológica. Recibió infusión de insulina y heparina, dieta estricta sin azúcar, sin fructosa, hipograsa con $10 \mathrm{~g}$ de lípidos, $300 \mathrm{~g}$ de carbohidratos complejos con 15-20 g de fibra dietética y $100 \mathrm{~g}$ de proteínas, y se cambió terapia farmacológica a fenofibrato $200 \mathrm{mg}$ /día, rosuvas- tatina $10 \mathrm{mg} /$ día, omega-3 $6 \mathrm{~g}$ al día y orlistat. Pese a todas las medidas implementadas, el paciente mantuvo hipertrigliceridemia hasta $3.276 \mathrm{mg} /$ dl por lo que se decidió inicio de aféresis. Luego de 5 sesiones, con volemia $3.600 \mathrm{ml}$ de recambio (albúmina 4\%) logró reducción de triglicéridos a $588 \mathrm{mg} / \mathrm{dl}$ ( $82 \%$ de reducción) y mantuvo niveles $<1.000 \mathrm{mg} / \mathrm{dl}$ con aféresis diarias (Figura 1A). Considerando que los episodios de pancreatitis previos fueron en contexto de triglicéridos $>1.000$ $\mathrm{mg} / \mathrm{dl}$, se establece como objetivo lograr reducción de niveles idealmente bajo dicho valor.

Posteriormente, se lograron distanciar sesiones de aféresis a 2 veces por semana. El paciente fue dado de alta y se acordó continuar aféresis crónica de manera ambulatoria por medio de una fístula arterio-venosa en su brazo izquierdo. Actualmente se encuentra con requerimiento de 1 plasmaféresis semanal (Figura 1B). Durante su evolución se intentó suspender estatinas, sin embargo, presentó incremento en triglicéridos séricos y episodios de dolor abdominal autolimitado sin cumplir criterios de PA, por lo que decidió reinicio de terapia previa. A la fecha, el paciente se mantiene en buenas condiciones generales, tolerando adecuadamente terapia y sin nuevos episodios de PA.

\section{Discusión}

El caso presentado probablemente corresponde al tipo de hipertrigliceridemia con trasfondo genético, considerando su severidad y descartando causas secundarias. La terapia médica se optimizó desde distintas aristas. En primer lugar, la restricción dietética de grasa es uno de los pilares fundamentales. Las recomendaciones incluyen reducir el consumo de grasa a $<20 \%$ del total de la ingesta calórica o incluso menos (10-15\%) y reducir el consumo de carbohidratos simples (como la fructosa $)^{12}$. En relación a la terapia farmacológica, se decidió manejo con varios fármacos sinérgicos con la finalidad de bloquear distintas vías de producción y metabolismo de triglicéridos. Se intentó retirar estatinas, sin embargo, tuvo alza sintomática de TG séricos en controles posteriores.

Durante años se han estudiado nuevos fármacos para el manejo de HTG severa. El año 2012 se aprobó Alipogene tiparvovec como terapia génica para pacientes con síndrome de quilomicronemia familiar (SQF). Se logró una reducción significativa de TG y del número de PA a las 3-6 semanas 
A. Inicio de aféresis Volemia $2.000 \mathrm{ml}$, albúmina $4 \%$

Cambio a volemia de $3.600 \mathrm{ml}$

a
\begin{tabular}{|l|c|c|c|c|c|c|c|c|}
\hline$N^{\circ}$ día & 1 & 2 & 3 & 4 & 5 & 6 & 7 & 8 \\
\hline TG pre & 3.276 & 1.203 & 1.025 & & & 674 & 737 & 1.232 \\
\hline TG post & 2.574 & 1.249 & & 1.257 & 588 & 139 & 351 & \\
\hline
\end{tabular}

B.

Cambio de modalidad

aféresis 1 vez por semana

\begin{tabular}{|l|c|c|c|c|c|c|}
\hline Año 2019 & Enero & Febrero & Marzo & Abril & Mayo & Junio \\
\hline TG (media) & 1.190 & 970 & 1.200 & 735 & 1.225 & 1.209 \\
\hline
\end{tabular}

\begin{tabular}{|l|c|}
\hline Año 2019 & Julio \\
\hline TG (media) & 1.477 \\
\hline Año 2020 & Enero \\
\hline TG (media) & 1.901 \\
\hline
\end{tabular}

Figura 1. A: Triglicéridos séricos luego del inicio de aféresis (mg/dl). B: Exámenes de seguimiento (media de triglicéridos séricos).

de tratamiento ${ }^{13}$. Desafortunadamente, se retiró del mercado el año 2017 por problemas comerciales. Pradigastat, un inhibidor de diacilglicerol O-aciltransferada 1 (DGAT1) que interviene en la síntesis de TG, redujo los niveles de TG entre $41-70 \%$, con la mayor parte de su actividad debido a una reducción de quilomicrones ${ }^{14}$. Otros target estudiados incluyen los péptidos simil a angiopoyetina 3 y 4 (ANGPTL3 y ANGPLT4), que actúan regulando el metabolismo de TG, y últimamente se ha desarrollado una nueva clase de drogas dirigidas a la inhibición de apoC3, con el fin de permitir el clearance de lipoproteínas ricas en TG. El estudio APPROACH ${ }^{15}$ evaluó la seguridad y eficacia de Volanesorsen (inhibidor antisentido del ARNm hepático de apoC3) en pacientes con SQF logrando una reducción en los niveles de TG a menos de $750 \mathrm{mg} / \mathrm{dl}$ en $77 \%$ de los pacientes. Parece una terapia promisoria para este tipo específico de pacientes.

Los mecanismos de acción de cada fármaco y el porcentaje relativo de reducción de triglicéridos se resumen en la Tabla 1.

El uso de plasmaféresis como tratamiento de la hipertrigliceridemia se utilizó por primera vez en $1978^{16}$ y desde entonces se ha empleado como una herramienta útil para el manejo de HTG severa. A la fecha, sólo se han publicado pequeños estudios y reportes de caso, la mayor parte en relación a complicaciones como $\mathrm{PA}^{17}$. El beneficio de esta terapia se atribuye a la reducción rápida de TG y a la remoción de proteasas del plasma. De acuerdo a la Sociedad Americana de Aféresis y la Asociación Americana de Medicina, la hipertrigliceridemia constituye una indicación clase III para el recambio plasmático terapéutico. En una serie, se logró reducción de hasta $70 \%$ de los niveles de triglicéridos luego de la primera sesión ${ }^{18}$. En nuestro caso, se logró una reducción de $82 \%$ en los niveles de triglicéridos séricos, sin embargo, requirió 5 sesiones para alcanzar niveles $<1.000 \mathrm{mg} / \mathrm{dl}$. 
Tabla 1. Estrategias farmacológicas para el manejo de la hipertrigliceridemia ${ }^{20}$

\begin{tabular}{|c|c|c|c|c|}
\hline & Mecanismo de acción & $\begin{array}{c}\text { Reducción TG } \\
(\%)\end{array}$ & Comentarios & $\begin{array}{l}\text { Tipo de } \\
\text { uso }\end{array}$ \\
\hline \multicolumn{5}{|c|}{ Terapias disponibles en la actualidad: } \\
\hline Fibratos & $\begin{array}{l}\text { Activación de PPAR- } \alpha \text {, } \\
\uparrow \text { niveles de } \mathrm{LPL}, \downarrow \text { ApoC3, } \downarrow \\
\text { síntesis hepática de TG por medio } \\
\text { de la inducción de oxidación de } \\
\text { AGL hepáticos y estimulación del } \\
\text { transporte reverso del colesterol }\end{array}$ & $30-50 \%$ & $\begin{array}{l}\text { Consideradas drogas de } \\
\text { primera línea. Lento inicio de } \\
\text { acción }\end{array}$ & $\begin{array}{l}\text { Agudo y } \\
\text { crónico }\end{array}$ \\
\hline Ácido nicotínico & $\begin{array}{l}\downarrow \text { lipólisis periférica vía HCAR3 } \\
\downarrow \text { secreción de VLDL }\end{array}$ & $10-30 \%$ & $\begin{array}{l}\text { Varios efectos adversos. Lento } \\
\text { inicio de acción }\end{array}$ & $\begin{array}{l}\text { Agudo y } \\
\text { crónico }\end{array}$ \\
\hline Estatinas & $\begin{array}{l}\text { Inhibición de la síntesis de } \\
\text { colesterol, } \uparrow \text { receptores de LDL }\end{array}$ & $\begin{array}{l}\text { Dosis moderada: } \\
\text { 10-15\% } \\
\text { Dosis alta: } \\
25-30 \% \\
\text { Combinación con } \\
\text { fibratos: } 50-60 \%\end{array}$ & $\begin{array}{l}\text { Usar en combinación con } \\
\text { otros fármacos para lograr } \\
\text { efecto sinérgico. Alto riesgo } \\
\text { de efectos adversos }\end{array}$ & $\begin{array}{l}\text { Agudo y } \\
\text { crónico }\end{array}$ \\
\hline Omega-3 & $\begin{array}{l}\downarrow \text { síntesis hepática de TG, } \\
\uparrow \text { b-oxidación peroxisomal, } \\
\uparrow \text { actividad y expresión de LPL en el } \\
\text { tejido adiposo, } \\
\downarrow \text { inflamación vía activación de } \\
\text { FFAR-4 }\end{array}$ & $\begin{array}{l}\text { 30-50\% con dosis } \\
\quad>4 \mathrm{~g} / \text { día }\end{array}$ & $\begin{array}{l}\text { Gran potencia farmacológica, } \\
\text { inicio de acción inmediato } \\
\text { Sin limitaciones para su uso }\end{array}$ & $\begin{array}{l}\text { Agudo y } \\
\text { crónico }\end{array}$ \\
\hline Orlistat & $\begin{array}{l}\downarrow \text { hidrólisis de TG en el intestino por } \\
\text { medio de la inhibición de la lipasa } \\
\text { gástrica }\end{array}$ & $30 \%$ & $\begin{array}{l}\text { Efectos adversos que pueden } \\
\text { condicionar adherencia } \\
\text { (urgencia fecal, deposiciones } \\
\text { oleosas, flatulencias) }\end{array}$ & $\begin{array}{l}\text { Agudo y } \\
\text { crónico }\end{array}$ \\
\hline MCT & $\begin{array}{l}\text { No induce síntesis ni formación de } \\
\text { quilomicrones. Induce b-oxidación } \\
\text { mitocrondrial de ácidos grasos }\end{array}$ & Variable & $\begin{array}{l}\text { Sin contraindicación } \\
\text { formal. Uso en pacientes } \\
\text { hospitalizados }\end{array}$ & $\begin{array}{l}\text { Agudo y } \\
\text { crónico }\end{array}$ \\
\hline Insulina & $\begin{array}{l}\text { Activación de LPL (acelera la } \\
\text { degradación de quilomicrones) }\end{array}$ & Variable & $\begin{array}{l}\text { Útil, especialmente en } \\
\text { pacientes diabéticos mal } \\
\text { controlados con HTG. Eficacia } \\
\text { limitada }\end{array}$ & Agudo \\
\hline Heparina & $\begin{array}{l}\text { Estimula la liberación de LPL } \\
\text { endotelial }\end{array}$ & Variable & $\begin{array}{l}\text { No recomendado como } \\
\text { monoterapia. Aumenta } \\
\text { la degradación de LPL y } \\
\text { depleción de depósitos } \\
\text { plasmáticos de LPL con el } \\
\text { riesgo de "rebote" luego de } \\
\text { inicio de tratamiento }\end{array}$ & Agudo \\
\hline \multicolumn{5}{|l|}{ Terapias en estudio: } \\
\hline $\begin{array}{l}\text { Alipogene tipar- } \\
\text { vovec }\end{array}$ & $\begin{array}{l}\text { Gen LPL exógena por medio de } \\
\text { vector viral. }\end{array}$ & $20-40 \%$ & $\begin{array}{l}\downarrow \text { Pancreatitis hasta } 50 \% \text {. } \\
\text { Retirado del mercado por sus } \\
\text { costos }\end{array}$ & Crónico \\
\hline Volanesorsen & $\begin{array}{l}\text { Inhibición de apoC3 permitiendo } \\
\text { clearance de lipoproteínas ricas en } \\
\text { TG }\end{array}$ & $56-86 \%$ & $\begin{array}{l}\text { Puede inducir } \\
\text { trombocitopenia y reacciones } \\
\text { cutáneas en sitio de inyección }\end{array}$ & Crónico \\
\hline Evinacumab & $\begin{array}{l}\text { Anticuerpo contra ANGPTL3 } \\
\text { (inhibidor de LPL) }\end{array}$ & $33-63 \%$ & $\begin{array}{l}\text { Puede producir reacciones } \\
\text { cutáneas en sitio de inyección }\end{array}$ & Crónico \\
\hline
\end{tabular}

PPAR- $\alpha$ : Peroxisome proliferator-activated receptor Alpha; LPL: Lipoproteinlipasa; ApoC3: Apolipoproteina C3; TG: Triglicéridos; AGL: Ácidos grasos libres; HCAR3: Hydroxycarboxylic acid receptor 3; VLDL: Very low density lipoprotein; LDL: Low density lipoprotein; FFAR4: Free fatty acid receptor 4; HTG: Hipertrigliceridemia; MCT: Triglicéridos de cadena media; ANGPLT3: Angiopoietin-like proteins 3 . 
Uso de plasmaféresis crónica ambulatoria en hipertrigliceridemia severa refractaria - C. Jure et al

Existe evidencia aún más limitada en relación al uso de plasmaféresis como tratamiento crónico. Un estudio caso-control ${ }^{19}$ evaluó el uso de plasmaféresis en 2 grupos: un grupo de pacientes con PA secundaria a HTG; y otro grupo de pacientes asintomáticos referidos desde atención ambulatoria con triglicéridos $5.000 \mathrm{mg} / \mathrm{dl}$ pero con historia de PA ("plasmaféresis profiláctica"). En el primer caso, se observó que la terapia condicionó una menor estadía hospitalaria e incidencia de complicaciones. En el segundo grupo (4 pacientes con plasmaféresis cada 3 meses y 2 pacientes con plasmaféresis cada 6 meses) luego de 2 años de seguimiento ninguno de los pacientes tuvo triglicéridos mayores a $5.000 \mathrm{mg} / \mathrm{dl}$ ni episodios de PA. En nuestro caso, el paciente se mantiene asintomático, manteniendo triglicéridos aceptables en comparación a sus valores previos, lo que apoyaría que en cierto grupo de pacientes con HTG severa, sin respuesta a medidas estándar, el recambio plasmático podría ser una opción factible como medida preventiva.

Cabe destacar el impacto positivo en la vida del paciente desde el inicio de la terapia, logrando mantenerse sin hospitalizaciones asociadas a complicaciones de HTG, mejorando su estado nutricional (IMC actual en $22 \mathrm{~kg} / \mathrm{m}^{2}$ ), y reinsertándose laboral y socialmente lo que finalmente constituye una mejoría en su calidad de vida.

Es de esperar que a futuro nuestro paciente pueda optar al uso de terapias farmacológicas con eficacia comprobada para distanciar y, eventualmente, suspender la terapia aferética. Para nuestro centro ha constituido un gran desafío al ser el primer hospital de la región con un paciente en plasmaféresis crónica realizada de manera ambulatoria, con aceptable respuesta a la fecha.

Agradecimientos: Este trabajo no habría sido posible sin el invaluable apoyo humano del Hospital San Pablo de Coquimbo. Muchas gracias por ayudarnos con este particular caso.

\section{Referencias}

1. Carroll MD, Lacher DA, Sorlie PD, Cleeman JI, Gordon DJ, Wolz M, et al. Trends in serum lipids and lipoproteins of adults, 1960-2002. JAMA 2005; 294: 1773-81.

2. Brahm A, Hegele RA. Hypertriglyceridemia. Nutrients 2013; 5: 981-1001.
3. Kolovou GD, Anagnostopoulou KK, Kostakou PM, Bilianou H, Mikhailidis DP. Primary and secondary hypertriglyceridaemia. Current Drug Targets 2009; 10 (4): 336-43.

4. Dron JS, Wang J, Cao H, McIntyre AD, Iacocca MA, Menard JR, et al. Severe hypertriglyceridemia is primarily polygenic. J Clin Lipidol 2019; 13 (1): 80-88.

5. Lewis GF, Xiao C, Hegele RA. Hypertriglyceridemia in the genomic era: a new paradigm. Endocr Rev 2015; 36 (1): 131-47.

6. Valdivieso P, Ramírez-Bueno A, Ewald N. Current knowledge of hypertriglyceridemic pancreatitis. Eur J Intern Med 2014; 25: 689-94.

7. Papachristou G, Machicado J, Stevens T. Acute Pancreatitis Patient Registry to Examine Novel Therapies in Clinical Experience (APPRENTICE): An international, multicenter consortium for the study of acute pancreatitis. Ann Gastroenterol 2017; 30: 106-13.

8. Whitcomb D. Acute pancreatitis. N Engl J Med 2006; 354: 2142-50.

9. Sandhu S, Al-Sarraf A, Taraboanta C. Incidence of pancreatitis, secondary causes, and treatment of patients referred to a specialty lipid clinic with severe hypertriglyceridemia: A retrospective cohort study. Lipids Health Dis 2011; 10: 157.

10. Yadav D, Pitchumoni CS. Issues in hyperlipidemic pancreatitis. J Clin Gastroenterol 2003; 36: 54-62.

11. Schwartz J, Padmanabhan A, Aqui N, Balogun R, Connelly-Smith L, Delaney M, et al. Guidelines on the Use of Therapeutic Apheresis in Clinical Practice-Evidence-Based Approach from the Writing Committee of the American Society for Apheresis: The Seventh Special Issue. J Clin Apher 2016; 31: 149-62.

12. Williams CM. Dietary interventions affecting chylomicron and chylomicron remnant clearance. Atherosclerosis 1998; 141 (1): S87-S92.

13. Gaudet D, Stroes ES, Methot J. Long-Term Retrospective Analysis of Gene Therapy with Alipogene Tiparvovec and Its Effect on Lipoprotein Lipase Deficiency Induced Pancreatitis. Hum Gene Ther 2016; 11: 916-25.

14. Meyers CD, Tremblay K, Amer A, Chen J, Jiang L, Gaudet D. Effect of the DGAT1inhibitor pradigastat on triglyceride and apoB48 levels in patients with familial chylomicronemia syndrome. Lipids Health Dis 2015; 14 (8): 1-9.

15. Witztum J, Gaudet D, Freedman S, Alexander V, Digenio A, Williams K, et al. Volanesorsen and Triglyceride Levels in Familial Chylomicronemia Syndrome. N Engl J Med 2019; 381: 531-42.

16. Betteridge DJ, Bakowski M, Taylor KG, Reckless JP, de Silva SR, Galton DJ. Treatment of severe diabetic 
hypertriglyceridemia by plasma exchange. Lancet 1978; 1 (8078): 1368.

17. Ewald N, Kloer HU. Treatment options for severe hypertriglyceridemia (SHTG): the role of apheresis. Clin Res Cardiol 2012; 7: 31-5.

18. Yeh JH, Chen JH, Chiu HC. Plasmapheresis for hyperlipidemic pancreatitis. J Clin Apher 2003; 18 (4): 1815.
19. Chang CT, Tsai TY, Liao HY, Chang CM, Jheng JS, Huang WH, et al. Double Filtration Plasma Apheresis Shortens Hospital Admission Duration of Patients With Severe Hypertriglyceridemia-Associated Acute Pancreatitis. Pancreas 2016; 45 (4): 606-12.

20. Ewald N, Hardt PD, Kloer HU. Severe hypertriglyceridemia and pancreatitis: presentation and management. Curr Opin Lipidol 2009; 20 (6): 497-504. 